\title{
Extensive Brown Tumors of Spine, Distal femur and Patella Presenting with Acute Cord Compression. A Case Report
}

\author{
TH Low, MS Orth (UM), LB Saw, MS Orth (UM), MK Kwan, MS Orth (UM), CrW Chan, MS Orth (UM), \\ AS Deepak*, FRCS \\ Department of Orthopaedic Surgery, University Malaya Medical Centre, Kuala Lumpur, Malaysia \\ *Prince Court Medical Centre, Kuala Lumpur, Malaysia
}

\begin{abstract}
We report a case of end-stage renal failure in a patient who presented with acute cord compression and extensive brown tumour involving the spine, distal femur and patella. Brown tumours of the long bone, spine and pelvis have been documented previously. However, the occurrence of brown tumour in sesamoid bones such as the patella is extremely rare and is indicative of widespread lesions and severe secondary hyperparathyroidism. These tumours typically respond poorly to medical treatment alone. The acute cord compression by spinal brown tumour necessitated urgent surgical decompression while the other lesions were treated with medical treatment and surgical parathyroidectomy.
\end{abstract}

Key Words:

Brown Tumour, Osteoclastoma, Renal Failure, Myelopathy

\section{INTRODUCTION}

Brown tumour is a cystic bone lesion classically associated with primary hyperparathyroidism, but it can also occur in end stage renal failure complicated by secondary hyperparathyroidism. The incidence of brown tumour in renal failure patients is been reported to be 1.5 to $13 \%$. Common sites affected are the mandible, ribs, pelvis and clavicle. Spinal lesion with cord compression and involvement of the extremities are uncommon while patella involvement is extremely rare. One reported case of brown tumour of patella was associated with primary hyperthyroidism ${ }^{1}$. We found no previous cases of brown tumour of the patella reported in association with secondary hyperthyroidism in renal failure patients. Here, we report a case in which a renal failure patient presented with acute cord compression and multiple brown tumours including a patella lesion.

\section{CASE REPORT}

A 40-year-old gentleman, with a 6-year history of haemodialysis for end-stage renal failure secondary to hypertensive nephropathy, presented with acute onset of lower thoracic spine pain with radicular pain towards the epigastrium along with bilateral lower extremity weakness for the previous week. Physical examination revealed lower thoracic spine tenderness with weakness of the lower limbs bilaterally. Muscle strength of the hip flexors and knee extensors were grade 4 and knee reflexes were brisk bilaterally. Sphincter tone was normal. During the admission process, he also complained of mild knee aches bilaterally. Plain radiographs of the spine revealed a lytic lesion at T11 with left pedicle destruction (Fig. 1A) and 'rugger-jersey' spine appearance (Fig. 1B). Plain radiographs of the hands showed subperiosteal erosion of phalanges. Plain radiographs of the knees showed a cystic lesion on distal metaphysis of the right femur and a multicystic lesion in the left patella (Fig. 2). Laboratory results showed a normal corrected calcium level $(2.6 \mathrm{mmol} / \mathrm{L})$ and an elevated phosphate level $(2.8 \mathrm{mmol} / \mathrm{L})$. Serum alkaline phosphatase was also elevated $(914 \mathrm{mmol} / \mathrm{L})$, as were parathyroid hormone levels $(222.0 \mathrm{pmol} / \mathrm{L})$. An urgent MRI disclosed multiple lesions at the T1, T11 and S1 (Figs. 3A and 3B). The lesion at T11 encroached into the spinal canal causing spinal cord compression (Fig. 3B). A percutaneous transpedicular bone biopsy was performed at T11. The specimen consisted of brownish tissue with histopathological findings of fibrous tissue with presence of multinucleated giant cells.

The spinal cord compression was treated with posterior laminectomy and instrumentation with insertion of a pedicle screw after embolisation of the T11 tumour to reduce tumour vascularity.

Postoperatively, back pain and radicular pain decreased and was accompanied by improvement in lower limb strength. However, the medical treatment failed to normalize the serum phosphate and parathyroid hormone level. A subtotal parathyroidectomy was performed four months later.

\section{DISCUSSION}

Brown tumours are cystic bone lesions formed by increased osteoclastic activity as seen in hyperparathyroidism. In chronic renal failure, phosphate retention and low serum 


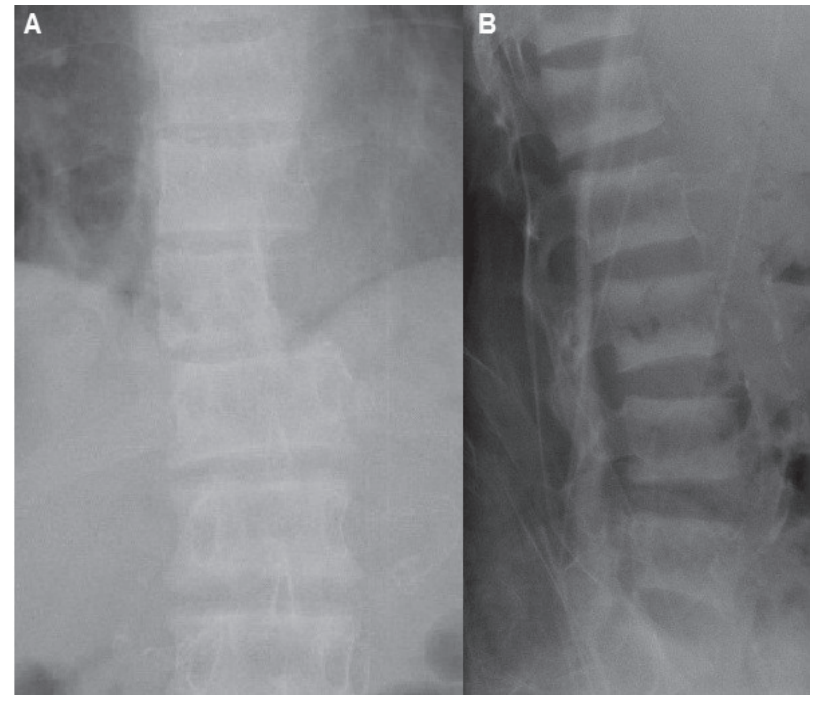

Fig. 1: Plain radiographs showing a missing left pedicle of T11 vertebra (A) and rugger-jersey appearance of lumbar spine (B).

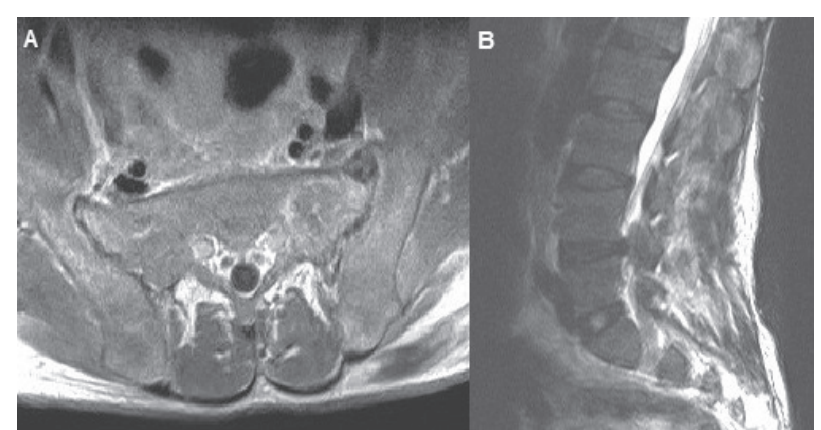

Fig. 3: (A) MRI (T1 weighted image) showing a brown tumour at the left sacral ala. (B) MRI (T2-weighted image) showing the collapse and retropulsion of T11 vertebra with spinal cord compression.

calcium due to the deficiency of 1(alpha), 25dihydroxycholecalciferol stimulates an increase in parathyroid hormone levels. Microfractures and haemorrhages commonly occur in these lesions due to their weak bony architecture. The resultant hemosiderin deposition gives the brown colour and hence the name of these tumours.

The diagnosis of brown tumour requires both the histological finding of fibrous tissue with multinucleated giant cells and the presence of hyperparathyroidism.

The definitive treatment of brown tumour is to normalize the serum calcium and phosphate level with calcium supplements and calcitriol, restrict dietary phosphate and phosphate binder intake. If these conservative treatments failed, total or subtotal parathyroidectomy should be performed. Success in normalizing serum calcium and phosphate then leads to remineralisation and resolution of lesions ${ }^{2,3}$

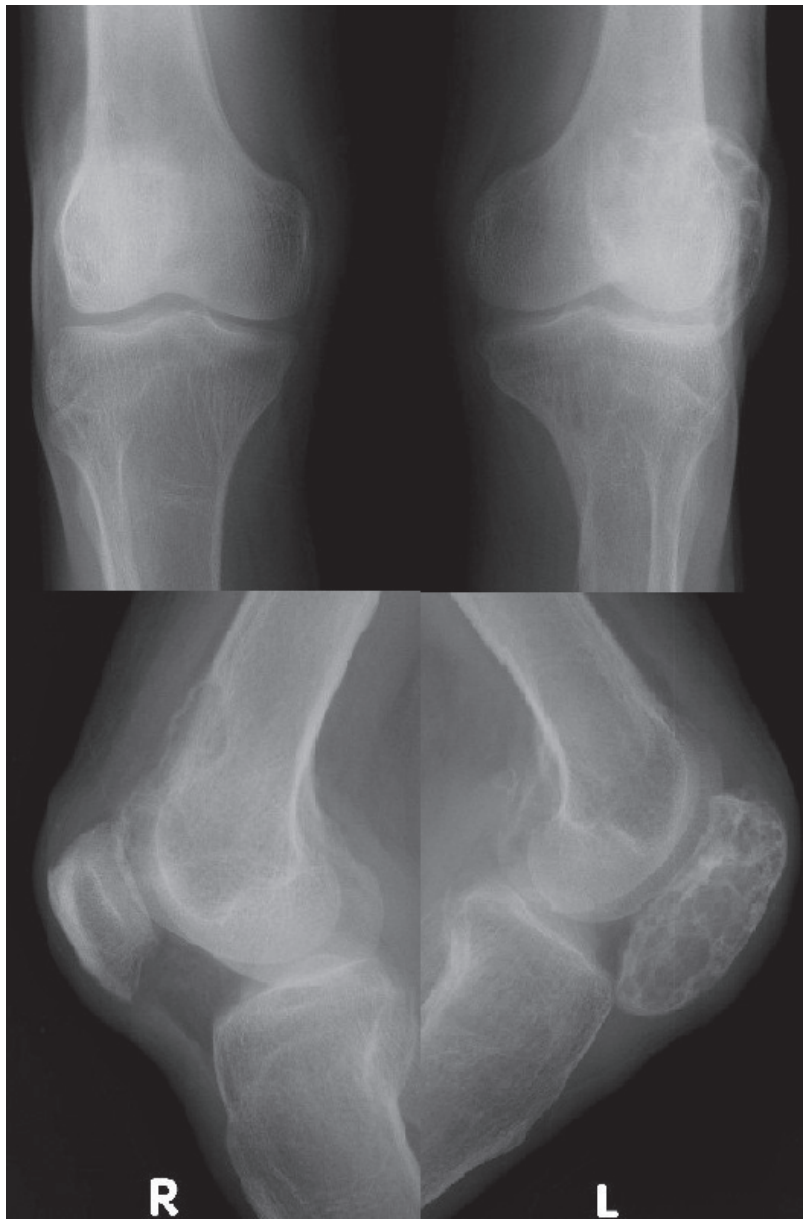

Fig. 2: Plain radiographs showing multiloculated lytic lesions in the left patella and right distal femur.

However due to acute spinal cord compression, the present case necessitated urgent posterior decompression to improve the chances of neurological recovery. Radical excision of the tumour was not required, as these tumours resolve with definitive treatment. Subsequently, measures to normalize serum calcium and phosphate were necessary to promote incorporation of the graft and to prevent expansion of other spinal lesions as well as to promote resolution of the nonspinal lesions ${ }^{3}$.

The incidence of brown tumour is expected to increase in line with the increased number of dialysis-dependent patients that have arisen due to improved medical treatment of renal failure and the lack of available organs for transplantation. A high index of suspicion of brown tumour should exist in end stage renal failure patients presenting with bony swelling, skeletal pain or acute neurological symptoms related to spinal lesions. The presence of brown tumours in sites such as the patella is rare and generally indicates that such lesions are widespread in the body thereby suggesting a severe stage of secondary hyperparathyroidism that may not respond to medical treatment alone. 


\section{REFERENCES}

1. Duran C, Ersoy C, Bolca N, Kiyici S, Yalcinkaya U, Erturk E, et al. Brown Tumors of the Maxillary Sinus and Patella in a Patient With Primary Hyperparathyroidism. Endocrinologist 2005; 15(6): 351-4.

2. Barlow IW, Archer IA. Brown tumor of the cervical spine. Spine 1993; 18: 936-7.

3. Vandenbussche E, Schmider L, Mutschler C, Man M, Jacquot C, Augereau B. Brown Tumor of the Spine and Progressive Paraplegia in a Hemodialysis Patient. Spine 2004; 29(12): 251-5. 